\title{
Probabilistic Foundations of Rabbinic Methods for Resolving Uncertainty
}

\author{
Moshe Koppel \\ Departament of Computer Science, \\ Bar-Ilan University, \\ Israel
}

email: moishk@gmail.com

\begin{abstract}
We consider several fundamental principles of rabbinic approaches to handling uncertainty for legal purposes. We find that nonnumerical versions of ideas subsequently developed in the literature on interpretations of probabilistic statements are useful for explicating these rabbinic principles.

Keywords: rabbinic law, interpretations of probability.
\end{abstract}

\section{Introduction}

The modern theory of probability is twice removed from Rabbinic laws concerning uncertainty. First, in its current form the theory of probability is simply the study of a particular class of functions and is not concerned with assigning probabilities to real-world events. Second, even if on the basis of certain stipulations, the theory is applied to actual events, it remains descriptive and not prescriptive. Nevertheless, certain philosophical issues which have arisen as a result of attempts to explicate the meanings of probabilistic statements are highly relevant to a proper understanding of Rabbinic approaches to uncertainty. In this paper, I will attempt to present a unified overview of Rabbinic laws concerning uncertainty. I will use ideas taken from the study of foundations of probability where these ideas seem helpful but will try to refrain from belaboring the analogy for its own sake.

One historical point needs to be emphasized. The modern theory of probability has its roots in the work of Pascal and others in the $17^{\text {th }}$ century. It would be utterly anachronistic to attribute to the $1^{\text {st }}$ century sages any foreknowledge of these developments. Moreover, doing so does not purchase any explanatory power with regard to Rabbinic approaches to uncertainty. At the same time, the claim that the ancients were bereft of any systematic thinking with regard to uncertainty is both arrogant and demonstrably false. I will use modern ideas about the foundations of probability as a starting point for identifying which probabilistic insights do and do not lie at the root of Rabbinic pronouncements on such matters.

Nevertheless, my approach in this article is unabashedly ahistorical: rather than chart a chronological progression of ideas or identify conflicting schools of thought, I will attempt to 
harmonize a broad range of sources. Where a Tann'ayitic or 'Amor'aic source permits multiple interpretations, I will not outline all views but rather select the most straightforward or consensual interpretation. Likewise, I will relate to the central ideas discussed in the vast post-Talmudic literature - both classical [2], [9] and contemporary [1], [3], [4], [6], [7], [10], [13] - devoted to Rabbinic laws concerning uncertainty but, for the sake of offering as straightforward and unified a treatment as possible, I will cite opinions of the commentators in an extremely selective manner. The fact that I marshal the support of a particular commentator regarding a particular point should in no way be taken to mean that I can claim such support regarding related points.

In the first part of this article, I will use the distinction between two types of majority principles - rub'a d'it'a qaman (literally: a majority which is in front of us) and rub'a d'leyt'a qaman (literally: a majority which is not in front of us) - to motivate a discussion of distinct definitions of probability. This will lay the groundwork for the explication of a number of thorny Rabbinic concepts involving uncertainty and indeterminacy.

\section{Interpretations of Probability}

The Talmud in Hullin 11a-11b interprets the phrase (Exodus 23:2) 'incline after the majority' ('aharey rabim l'hatot) to mean that decisions of a court are decided by majority. This is then generalized to the above-mentioned principle of rub'a d'it'a qaman (henceforth: RDIK), which includes other cases such as that of 'nine stores,' i.e., a piece of meat is found in the street and all that is known is that it comes from one of ten stores, nine of which sell košer meat. In such cases we apply the principle that 'that which is removed, was removed from the majority' (kol d'pariš merub'a parišs; henceforth: pariš). The Talmud states that this inference covers only the principle of $R D I K$, of which Sanhedrin and 'nine stores' are offered as typical examples, but not the parallel principle of rub'a d'leyta qaman (henceforth: RDLK). The Talmud offers a number of examples of $R D L K$ where the majority is followed because it would be impossible to function normally or adjudicate cases without doing so (but concludes that precisely because of that impossibility these cases can't serve as a basis from which to infer a general principle of $R D L K$ ). Several cases of $R D L K$ that are illustrative are that the husband of one's mother (at the time of conception) may be presumed to be one's father, that a child may be presumed to be potentially fertile and that a murder victim may be presumed not to have been suffering from a prior life-threatening condition.

What is the precise difference between $R D I K$ and $R D L K$ ? Although the names are suggestive, the Talmud offers no explicit definition of $R D I K$ and $R D L K$ and no rationale for treating them differently. We might, however, shed considerable light on the distinction by considering an interesting philosophical debate dating back to the 1920's which covers similar conceptual territory. The rest of this section will consist of a slightly lengthy diversion through that territory.

Let's consider carefully what exactly we mean when we say that the probability of some event is $p / q$. Early (the $17^{\text {th }}$ and $18^{\text {th }}$ century) work in probability was motivated to a large extent by games of chance (coins, cards, dice). Thus, when somebody said that 'the probability of the event $H$ is $p / q$ ' it was understood that what was meant was that the event $H$ obtained in $p$ out of $q$ equally likely possible outcomes. Thus, for example, when we say the probability that the sum of two throws of a die will be exactly six is $5 / 36$, we mean that there are 36 equally likely possible throws and 5 of them have the desired property. Similarly, in the case of the found meat, there are ten possible sources for the meat and nine of them are košer, so we might say that the probability that the meat is košer is $9 / 10$. This understanding of probabilistic statements is usually called the 'classical' interpretation [5].

What is interesting for our purposes is that the classical interpretation turns out to be inadequate as a definition of probability. This became obvious once insurance companies began using probability theory to compute actuarial tables. What does it mean to say that 'the probability that a healthy forty-year-old man will live to the age of 70 is $p / q$ '? What are the q equally likely possible outcomes, $p$ of which find our insurance policy holder celebrating his seventieth birthday? No such thing. This led philosophers such as Reichenbach [8] and von Mises [11] to suggest the 
'frequentist' interpretation of probability: the statement that 'the probability that a healthy fortyyear-old man will live to the age of 70 is $p / q$ ' means that of the potentially infinite class of hypothetical healthy forty-year-old men, the proportion who will see seventy is $p / q$.

It is important to understand that according to each of these interpretations, the classical and the frequentist, there is always some subjective aspect in assigning a probability to an event. In the case of classical probability, this subjective element is rather benign: we need to define the underlying 'equally likely' cases, or what is called in formal parlance, the 'sample space.' For example, in the case of 'nine stores,' we might just as plausibly use as our sample space the three shopping malls in which the stores are concentrated or perhaps the ten thousand pieces of meat that are unequally distributed among the stores. The choice of which sample space is most appropriate is ultimately a matter that must simply be stipulated. It is tempting to imagine that the 'right' sample space is the one in which the various elements are equally probable. But obviously, this formulation is circular since it is the very notion of probability that we are trying to define. To be sure, in many cases, there is a rather obvious first choice of sample space. For example, in tossing a die, we would naturally identify the six possible faces as our sample space. This intuition rests on some sort of 'indifference principle' (why should one face be more likely than another?). But such indifference principles have proved remarkably resistant to precise formulation. Ultimately, the assignment of sample space is a matter of stipulation.

If in the case of classical probability, assigning a probability to an event requires a bit of judgment, in the case of frequentist probability such an assignment is fraught with judgment. Think of the example in which we wish to determine the probability that a particular child is potentially fertile (actually in the situation described in the Talmud we wish only to determine that this probability is greater than $1 / 2$ ). We wish to do so by invoking some rule that says: there is some reference class $A$ in which this child is a member and the expected proportion of members of $A$ which are potentially fertile is $p / q$. This expected frequency is in turn determined by our past experience with members of class $A$ and the frequency of fertility they exhibited. But what class $A$ is appropriate? Should $A$ be the class of all young mammals or all human children or perhaps the class of all children who share this child's medical history or the class of children who share this child's medical history and genetic stock? If we define the class too broadly we run the risk that our experience with the class is irrelevant to the particular child in question. If we define it too narrowly we run the risk that our experience with the class is too limited to provide any reliable information with regard to the class in general. And if we define it bizarrely (say, the class consisting of this child and all major household appliances), the results are, well, bizarre. The selection of the reference class $A$ as well as the determination that our experience with samples from that class is sufficient to project some statistical law onto the whole class are matters of judgment.

Consider now the extreme case of a probabilistic statement such as 'the probability that the United States will attack Iraq within two months is $60 \%$.' The problem with such statements is that the events in question belong to no natural class since the ensemble of relevant facts renders the case unique. It is implausible that we mean to say that in $60 \%$ of cases like this an attack occurs, because there aren't any cases quite 'like this.' Since according to the frequentist interpretation every probabilistic statement must refer to some class, these statements are utterly meaningless within the frequentist framework and indeed are rejected as such by von Mises and others.

One attempt to salvage such statements as meaningful has involved yet another interpretation of probability, the 'subjectivist' interpretation. According to this interpretation, the statement that the probability of some event is $p / q$ is taken to reflect the degree of certainty with which some rational observer is convinced of the correctness of the statement, as might be reflected in a betting strategy. Unlike the previous interpretations, such an interpretation does not require the identification of any relevant class. For example, for someone to say that the probability that the United States will attack Iraq within two months is $60 \%$ is simply to say that they regard as fair either side of a bet with 3:2 odds in favor of such an attack occurring.

To summarize, there are at least three different kinds of probabilistic statements: classical, frequentist and subjective. For each type, any instance of such a statement is meaningful only to the 
extent that at least one potentially fuzzy factor can be plausibly defined. In the classical case this factor is a sample set, in the frequentist case it is a reference class, and in the subjectivist case it is simply the strength of a hunch.

In the following sections, we will see how various Rabbinic methods can be best understood in relation to these different types of probabilistic statements. Moreover, we will see that different ways of resolving the fuzzy aspects of probabilistic statements can neatly account for certain apparent anomalies. In the next section, we will explain differences between the conditions and consequences of $R D I K$, on the one hand, and those of $R D L K$, on the other. After that we will clarify when $R D I K$ is applied and when a converse rule (qavu'a) is applied and will elucidate the difference between uncertainty (safeq) and indeterminacy.

\section{Rub'a d'itt'a qaman and rub'a d'leyt'a qaman}

We will define the principle of $R D I K$ more precisely in the next section but for now it is enough to define it roughly as follows: A random object taken from a set a majority of the members of which have property $P$, may be presumed to have property $P$. As so defined, the principle does not require any (but perhaps the most naive) probabilistic notions. Nevertheless, it is evident that the classical interpretation is fully adequate for a probabilistic formulation of $R D I K$ : RDIK amounts to specifying the members of the set as a sample space and following the result with probability greater than $1 / 2$. Note that $R D I K$ refers specifically to a set of q concrete objects, $p$ of which have some property, while the classical definition of probability refers more generally to $q$ possible outcomes (which may be abstract).

The classical interpretation is, however, clearly irrelevant to the examples of $R D L K$ we have seen. The frequentist interpretation, on the other hand, squares with $R D L K$ perfectly [7]. Simply put, all examples of $R D L K$ are statistical laws: most children born to married women are fathered by their husbands, most children are ultimately fertile, most people are not about to die, etc.

The identification of $R D I K$ with the classical interpretation and $R D L K$ with the frequentist interpretation will help us clear up a number of difficulties, as we shall see presently. We should note in advance, however, that the case should not be overstated. While the Rabbis certainly distinguished between two distinct kinds of majority that can be neatly embedded in full-blown theories of numerically quantifiable probability, it does not follow - and we are not suggesting that the Rabbis were in conscious possession of any such theory.

Let us begin with the question of which is stronger, $R D L K$ or RDIK. Later commentators have marshaled proofs for each possibility, the most salient of which follow.

The strength of $R D L K$ relative to $R D I K$ can be clearly seen in the following: It is wellestablished that we do not convict in capital cases based on mere likelihood (Babylonian Talmud, Sanhedrin 38a). Thus, consider the case of an abandoned baby boy, called an 'assufi, whose mother is one of a given set of women one of whom is a non-Jew. In this case, there is a RDIK in favor of the child's Jewish maternity. While such a child may be regarded as a Jew for certain purposes, a woman who eventually marries him cannot be convicted of adultery, since 'we do not administer the death penalty on the basis of uncertainty' (Maimonides, Hil. Issurei Biah 15:27). Nevertheless, consider another case of uncertain maternity, in which a woman has a relationship with a child that is typical of that of mother and son but, as is generally the case, there are no witnesses to the birth. In this case, there is a $R D L K$ in favor of the woman's maternity. If she and the 'son' are witnessed having sexual relations, they can be convicted for incest, since 'we administer the death penalty on the basis of presumptions' (Maimonides, Hil. Issurei Biah 1:20). Clearly, RDLK in these cases is stronger than $R D I K$.

In other cases, however, the weakness of $R D L K$ relative to $R D I K$ is evident. For example, the Babylonian Talmud (Yevamot 119b) cites the view of Rabbi Meir that a majority-based argument generally does not trump even a mere contrary status quo. Thus, for example, dough of tithes that was last known to be ritually clean but was found in the proximity of a child who is contaminated cannot be burned (as would ordinarily be done for contaminated tithes), according to 
Rabbi Meir, on the basis of a $R D L K$ that children typically pick at dough in their vicinity (Babylonian Talmud, Qiddušin 80a), since this (RDLK-type) majority argument is inadequate to overcome the status quo of the dough being clean. The Tosafists (Yevamot 67b s.v. 'ein hošešin, Yevamot 119a s.v. kegon) marshal proofs that this principle holds only with regard to $R D L K$, but that $R D I K$ always trumps a status quo presumption. Moreover, according to R. Yochanan, in the case of the dough even the Rabbis who disagree with R. Meir would concede that the dough can't be burned on the basis of this $R D L K$. Nevertheless, they would not so concede in a case of $R D I K$ (see Babylonian Talmud, Qiddušin 80a and the gloss of Rashi s.v. im rov). Thus, in these cases RDLK is weaker than $R D I K$.

We might be able to reach a definitive answer regarding which is stronger, $R D I K$ or $R D L K$, by explaining away one or the other set of proofs. But to do so would be to answer the wrong question. To understand the crucial difference between $R D I K$ and $R D L K$, let's recall the difference between the classical interpretation of probability and the frequentist interpretation.

In the case of classical probability, the part that is left to judgment is rather limited. Typically, a rather straightforward sample space is taken for granted. Once that's taken care of, assigning a probability is a simple matter of calculation. In fact, in the limited case of $R D I K$, the cases need only be counted. In the case of frequentist probability, however, selecting a reference class and then estimating frequencies within the class requires a substantial investment of judgment. With what confidence can we assert that for some class A the event in question occurs with some sufficiently high frequency? Answering this question, even loosely, is inevitably a matter of judgment. Hence, $R D L K$ can only be established based on rabbinic judgment.

Consequently, if you've seen one RDIK you've seen them all - unless there is some countervailing principle that prevents its application, $R D I K$ is a decision procedure that resolves, but does not dispel, uncertainty in favor of the majority regardless of whether $p / q$ is .99 or .51 . That is, in applying the principle of $R D I K$ we acknowledge that there is uncertainty but the $R D I K$ allows us to decide in favor of the majority much in the way that a majority vote settles a case in court. Invoking $R D I K$ is not sufficient, however, to achieve the degree of certainty necessary to establish the facts of a capital case.

Unlike $R D I K$, however, there are various types of $R D L K$. The apparent contradiction regarding the relative strengths of $R D I K$ and $R D L K$ simply reflects the fact that different applications of $R D L K$ have different strengths (both in terms of the strengths of the laws themselves and in terms of the strength of the evidence for the laws). Since $R D L K$ is always a product of rabbinic judgment, it stands to reason that they exercise this judgment variably. There are three types of decision rules and, depending on rabbinic judgment, $R D L K$ can be any one of them.

The middle type is the one we have seen in the case of $R D I K$ - a resolution procedure. These are often referred to as 'hakra'ah.' (This term, as well as the parallel terms below, was first proposed in [9].) An example of this is the $R D L K$ that most births are not of healthy males (Babylonian Talmud, Hullin 77b).

There are stronger decision rules which simply render irrelevant the minority possibility some examples of $R D L K$ are treated as certainties in the sense that we proceed as if the uncertainty has not simply been resolved but rather has been dispelled altogether. These are often referred to as 'beirur'. It is about these that we say 'we administer the death penalty on the basis of presumptions' - in capital cases certainty is required and these examples of $R D L K$, unlike any example of $R D I K$, do indeed provide certainty for legal purposes (at least regarding the establishment of relevant background facts [2, section 4:8]; tying a defendant to a particular act requires witnesses).

Finally, there are weaker decision rules that are merely 'defaults' in the sense that they are applied only as last-resort tie-breakers when no more substantive decision rule is available. These are often referred to as 'hanhagah.' The typical example of a default rule in rabbinic law is a status quo argument. In some cases, $R D L K$ is established merely as a default rule so that at most it can neutralize, but not defeat, another default rule such as status quo. For R. Meir, most cases of $R D L K$ are of this variety. 


\section{Rub'a d'itt'a qaman and qavu'a}

Let's now return to the principle of $R D I K$ and attempt to define it more precisely. We have already seen that according to the Talmud (Babylonian Talmud, Hullin 11a), this principle covers both the case of majority vote in Sanhedrin and that of 'nine stores' where the meat is found on the street. Moreover, the Talmud often invokes the related, though clearly not identical, principle that a mixture of permitted and forbidden objects may sometimes be assigned the status of the majority (bitul b'rov).

The generalization from the case of majority vote to cases such as 'nine stores' is not inevitable - the case of voting is more a procedural issue than one of resolving uncertainty. According to Talmudic principles [12, section 5:7], if a prophet declared the questionable piece of meat to have come from the minority, his claim might be decisive, but if he ruled in accord with the minority position in the Sanhedrin, we would ignore him (Babylonian Talmud, Bab'a Mezy 'a' 59b). Similarly, it has been argued [2, section 3:4] that the extension to bitul b'rov is not inevitable, as the Talmud seems to assume. Clearly, the Talmud is operating with a majority principle sufficiently general to cover all of the above cases.

Before we consider what this principle might be, let's consider the remarkably similar situation with regard to another decision principle, namely, that 'that which is fixed is as half and half' (kol qavu'a k'mehzah al mehzah dami; henceforth: qavu'a). Like RDIK, the case identified in the Talmud as the 'source' case of qavu'a is a procedural matter. Someone throws a stone into an assembly of nine Israelites and one Canaanite, intending to kill whichever person the stone happens to hit. The question is whether this unspecific intention is sufficient intention to kill an Israelite to warrant conviction for murder of an Israelite (a distinct offense from that of killing a Canaanite). The Rabbis apply the principle of qavu'a to determine that the Israelite majority does not render the intention sufficient (Babylonian Talmud, Ketubot 15a). What exactly the principle might be requires explanation. But note that in this case there is no doubt that the actual victim was indeed an Israelite and not a Canaanite. The issue under discussion is only whether the intention to kill 'some member of this group' can be regarded as the intention to kill an Israelite. Thus, there is no uncertainty regarding any of the facts of this case and no decision-method for resolving empirical uncertainty is called for.

The Talmud then cites as the classic example of qavu'a, the parallel case to that of 'nine stores' that we considered above: 'If there are nine stores which sell košer meat and one which sells non-košer meat and someone took [meat] from one of them but he doesn't know from which one he took, the meat is forbidden.'

The parallelism between RDIK and qavu'a is remarkable. In both, the 'source' case involves court procedures and includes no elements of actual uncertainty and in both the standard case is a version of 'nine stores' in which the central issue is apparently one of uncertainty. This suggests that $R D I K$ and qavu'a do not directly concern uncertainty, but rather are dual principles regarding mixed sets which cover cases of uncertainty as a by-product.

The principle of $R D I K$ might thus be formulated this way:

Given a set of objects the majority of which have the property $P$ and the rest of which have the property not- $P$, we may, under certain circumstances, regard the set itself and/or any object in the set as having property $P$.

The principle of qavu'a is the opposite of this:

Given a set of objects some of which have the property $P$ and the rest of which have the property not- $P$, we may, under certain circumstances, regard the set itself, and consequently any object in the set, as being neither $P$ nor not- $P$ but rather a third status. We can call this status hybrid, or perhaps, indeterminate. 
It is important to note that $R D I K$ comes in two varieties: $R D I K$ can assign a single status to the entire mixed set (as in the case of bitul b'rov) or it might assign a status directly to an individual object in the set (as in pariš). Qavu'a, on the other hand, comes in only one variety: a hybrid status must be assigned to a set and then only indirectly to an individual item in the set. When qavu'a is invoked, each individual item in the set loses its individual identity and is regarded simply as a fragment of an irreducibly mixed entity. It is not treated as an individual of uncertain status but rather as a part of a set that is certainly mixed. Given this, we are ready to answer the central question: When do we apply RDIK and when do we apply qavu'a?

Roughly speaking, the idea is that when an object is being judged in isolation, it must be assigned a status appropriate to an individual object; when it is judged only as part of a set, it can be assigned some new status. Qavu'a can only be invoked in the latter case. To see this distinction very starkly, consider two scenarios in each of which we have before us a box containing nine white balls and one black ball.

Scenario 1: I reach into the box, pull out one ball without showing it to you and ask: What is the color of this ball?

Scenario 2: I don't reach into the box, but instead ask: What is the color of a random ball in this box?

In the first case, if you were to answer, say, 'black,' your answer would be either true or false, but either way would be an appropriate response to the question that was asked. There is a determinate answer to the question, although this answer is unknown to you. In the second case, the answer 'black' (or 'white') is neither true nor false, since there is no determinate answer to the question. You could say nothing more specific than that the box contains both white and black balls.

Obviously, the case of the stone-thrower considered above is analogous to scenario 2 asking about the status of an unspecified member of the group is like asking about the color of an unspecified ball. The appropriate level at which to assign status in this case is the level of the set, not the level of the individual, and the set is indeed mixed. This is the sort of case in which qavu'a can be invoked.

By contrast, a piece of meat that is found in the street is clearly analogous to scenario 1 - the status of a particular item is in question. This is the kind of case in which RDIK is invoked. Admittedly, the case of a piece of meat bought in one of the stores might plausibly be regarded as analogous to scenario 1 since the act of buying could be considered analogous to pulling out a specific ball. However, the Rabbinic principle is, somewhat counter-intuitively, otherwise: apparently, the critical moment is the one prior to actually encountering the piece in question. When the piece is found on the street, it is judged as an individual because prior to the moment that it is found, it is already no longer 'in the set.' When the piece of meat in question is bought in the store, prior to its being bought it is indeed 'in the set.'

The distinction between qavu' $a$ and RDIK might be restated in terms of the issue of sample space selection considered above. RDIK assumes the "standard" sample space. In the case of the meat found in the street, that sample space is the set of stores. But qavu'a entails the selection of a non-standard, but entirely sensible, sample space: the single element consisting of the entire set of stores. This single item is mixed.

Let us now spell out in detail the precise method for determining when to apply $R D I K$ and when to apply qavu'a.

First, there are a number of cases in which qavu'a cannot be invoked because a hybrid status is inappropriate. decision.

In the case of a vote in Sanhedrin which is, by definition, a mechanism for rendering a

If uncertainty regarding the status of an individual object that belonged to the set arose only after the object had been isolated from the set (pariš), then it is this object alone that must be 
assigned some status. While a member of a set consisting of objects some of which are $P$ and some of which are not- $P$ can be assigned a hybrid status as part of the set, an individual object being assigned a status on its own cannot. Thus, we need to choose either $P$ or not- $P$ for this object and we choose the majority of the set from which it comes. For example, in the case of 'nine stores' in which the meat is found on the street, the isolated piece of meat is assigned either the status ' $k o s ̌ e r '$ or the status 'non-košer.'

Similarly, if the set is somehow 'incohesive,' so that each object in it is regarded as having left the set, we apply RDIK and not qavu'a. Thus, for example, a set of travelers passing through a town do not constitute a set for purposes of qavu'a, while the residents of the town do (Babylonian Talmud, Ketubot 15b; Babylonian Talmud, Yom'a 84b).

Finally, if it is not certain that the set contains any objects that are, say, not- $P$, the set cannot be assigned a hybrid status (formally, it is said to lack the necessary condition of 'ithazeq 'issur'a) and RDIK is invoked rather than qavu'a. Thus, the Toseft' $a$ (Taharot 6:3) considers a case in which we are given a mixture of ten loaves, including one loaf that is ritually unclean, that is eaten in two rounds of five loaves each. Those who eat in the first round are rendered ritually unclean because at that point the set certainly contains one unclean loaf, but those in the second round are not unclean because by then the set might not contain an unclean loaf.

To summarize: in all cases in which we are not assigning a status to a mixed set, qavu'a is not invoked but rather $R D I K$. Note that although in these cases the membership of the doubtful item in the set, or the cohesiveness of the set itself, may be inadequate for invoking qavu'a, this does not diminish the relevance of the set for purposes of RDIK. Thus, for example, even though the piece of meat found on the street cannot be assigned a hybrid status because it is not part of the set, the fact that the meat is known to have originated in the set still renders the composition of the set (i.e., the majority) relevant to determining the status of the piece.

When the above rule does not apply (that is, the issue is the status of a mixed set), there are cases in which $R D I K$ is not applicable. In particular, for the case of a mixed set we cannot invoke RDIK whenever bitul b'rov is not possible.

First, if the objects in the set are each identifiable as either $P$ or not- $P$ (nikar bimkomo). For example, in "nine stores" the status of each store is known, it is only the origin of a particular piece of meat that is in doubt. Clearly, in such a case, we can't define the set as either $P$ or as not- $P$; as a set, it is both.

Second, if individual objects in the set are each regarded as sufficiently significant that the status of each cannot be subordinated to the status of the set or if bitul b'rov is inapplicable for any other reason. Thus, given a herd of oxen including one that has been sentenced to death and is forbidden for use, we can't invoke bitul b'rov due to the significance of living creatures and hence we invoke qavu'a by default (Babylonian Talmud, Zevahim 73b).

Third, if the set includes an equal number of objects that are $P$ as are not- $P$. In such a case, bitul b'rov is obviously not possible.

In all the above cases, the set fails to take on a single status as a set and hence the principle of qavu'a can be invoked: the set is assigned a new hybrid status ( $P$ and not $P$ ) as are individual objects drawn from the set.

Finally, if neither of the above rules apply (so that we have a mixed set where bitul b'rov is possible), $R D I K$ is invoked. This is the ordinary case of bitul b'rov. It is important to note that, as in the case of qavu'a, bitul b'rov applies when the set is being judged as a set. Bitul b'rov is simply applied prior to qavu'a. Thus, by the time qavu'a is considered the set is no longer a mixed set but rather a uniform set. 


\section{Qavu'a and safeq}

The crucial distinction between uncertainty (safeq) regarding an individual object and qavu'a, which is a definite hybrid status assigned to a set, cannot be over-emphasized. When qavu'a is invoked, it is the definite mixed status of the entire set that concerns us and not the uncertain status of any individual item in the set. It is generally the failure to appreciate this distinction that leads to the conclusion that qavu'a is completely counter-intuitive.

Let's consider for a moment the alternative, more common, explication of qavu'a as merely a leveling of the playing field in which the case is treated as a symmetric safeq. On this understanding, which I reject, the sample space would contain two elements: košer and non-košer. According to my explanation, in cases of qavu'a, the sample space consists of a single element: the entire mixed set. Might not the phrasing "that which is fixed (qavu'a) is as half and half" suggest that the rule is in fact that we assign each status a probability of $1 / 2$, that is, that we have a sample space consisting of two elements? Why do I reject this possibility?

First of all, because such a rule would be arbitrary and the one I argue for is perfectly sensible. Moreover, the notion that 'half and half' refers to a probability of $1 / 2$ is utterly anachronistic. The assignment of probabilities to the range $[0,1]$, so that $1 / 2$ is in the middle, is a relatively recent convention. The phrase 'half and half' refers rather to set composition and not to probability. Specifically, it refers to the third case in Rule 2 of the qavu'a/RDIK rules above in which qavu' $a$ applies to a mixed set that includes an equal number of objects that are $P$ as are not- $P$. The point of the rule that 'that which is fixed (qavu'a) is as half and half' is that in all cases that satisfy the conditions for qavu'a, RDIK is not invoked just as it is obviously not invoked in the case where there is no majority.

Finally, there are important halakhic differences between cases which are deemed safeq and cases where qavu' $a$ is applied. For example, if a person had before him two indistinguishable pieces of meat, one košer and one non-košer - a case of qavu'a - and he ate one of them, he is obligated to bring a special sacrifice known as asham taluy. But if he had before him one piece, possibly košer but possibly non-košer - a case of safeq - he is not so obligated (see Maimonides, Hil. Šegagot 8:2). Similarly, if a mouse takes a piece from a mixed pile of pieces of leaven and of matzah, in a manner such that the principle of qavu'a would apply, into a house which has been inspected for Passover, the house must be re-inspected. But if it took a single piece of which has an even chance of being leaven or matzah into the house - this is a safeq - the house need not be re-inspected (see Maimonides, Hil. Hamez u-Mazah 2:10-11). In the case of safeq, we can presume that an inspected house remains free of leaven since one possible resolution of the uncertainty regarding the subsequent events is consistent with this presumption. In the case of qavu'a, however, there is no uncertainty to resolve. Rather, some object of known mixed status has certainly been brought into the house; this is enough to nullify the presumption.

Now that we have established that cases of qavu'a are not cases of safeq, which cases are in fact safeq? The status of an object is safeq when it is not judged as part of a set (so that qavu'a and bitul b'rov do not apply) and it has not been removed from a set with a majority (so that pariš does not apply) and it does not belong to some reference class for which some statistical law is known (so that $R D L K$ does not apply). A simple example of safeq is one in which a piece of meat is found in the street and might have come from one of two stores, one košer and one non-košer.

In such cases, second-order default rules might be invoked to determine a course of action. These second-order rules involve the nature and severity of the prohibition in question and relevant presumptions, a detailed discussion of which is beyond the scope of this article.

\section{Acknowledgement}

This paper is a revised and abridged version of M. Koppel, Resolving uncertainty: a unified overview of rabbinic methods, Tradition, 37, 2003, pp. 27-51. 


\section{References}

1. Beck, J. Shtern, V. The Talmudic concepts of making decisions under uncertainty, $B D D, 15$, 2004, pp. 37-64.

2. Heller, A. L. Shev Shmaat'ta (in Hebrew), Lemberg: Rapaport, 1804.

3. Koppel, M. Inclusion and Exclusion (in Hebrew), Higayon, 1, 1989, pp. 9-11.

4. Koppel, M. Further comments on rov and qavu'a (in Hebrew), Higayon, 4, 1997, pp. 49-52.

5. Laplace, P. S. A Philosophical Essay on Probabilities, transl. from French $6^{\text {th }}$ ed. [1840], London: Wiley, 1902.

6. Moscovitz, L. On the principles of majority (rov) and ithazeq isura (in Hebrew), Higayon, 4, 1997, pp. 18-48.

7. Rabinovitch, Nachum L. Probability and Statistical Inference in Ancient and Medieval Jewish Literature, Toronto: Univ. of Toronto Press, 1973.

8. Reichenbach, H. The Theory of Probability, Berkeley: University of California Press, 1949.

9. Shkop, S. Sha'arei Yosher, Warsaw: Hutner, 1928.

10. Taylor, N. The definition of rov in rabbinic law (in Hebrew), Higayon, 4, 1997, pp. 53-65.

11. Von Mises, R. Probability, Statistics and Truth, New York: Dover, 1957.

12. Wasserman, E. Qunteres Divrei Soferim, Pietrikov: Folman, 1924.

13. Werblowsky, Y. Rov and probability, Higayon, 4, 1997, pp. 5-22. 